\title{
Development of spring wheat lines with a reduced period from germination to heading using the marker-assisted selection
}

\author{
Stasyuk A.I.*, Kiseleva A.A., Salina E.A. \\ Institute of Cytology and Genetics, SB RAS, Novosibirsk, Russia \\ *e-mail: stasyuk@bionet.nsc.ru
}

The time of heading of wheat is an important factor that determine the adaptation of plants to environmental conditions. The heading time of spring wheat is determined by genes that control the plant reaction to vernalizing temperatures ( $V R N-$ vernalization genes), and photoperiod sensitivity (PPD1 - photoperiod response genes). The different combinations of alleles of these genes result in different heading time. Marker-assisted selection enables to accelerate the development of spring wheat forms, differing in heading time. Using the results of the analysis of the allelic variation of the $V R N$ and $P P D 1$ genes in Siberian spring wheat varieties, we selected the early Tulun 15 variety, with photoperiod insensitive $P p d-D l a$ and rare $V r n-B 3 a$ allele, associated with early flowering. The cultivar Tulun 15 was crossed with the middle early cultivar Obskaya 2. Using allele-specific primers $\mathrm{F}_{2}$ generation plants with different combinations of $V R N$ and PPD1 alleles were detected. These plants were divided into four haplotypes. The $\mathrm{F}_{3}$ generation plants resulted from self-pollination of these plants were sown in the field to study the effect of the haplotypes on the heading time. The time from germination to heading of the plants with different haplotypes was as follows: haplotype 1 ( $\mathrm{Vrn}$-Ala Vrn-B1c vrn-D1 Vrn-B3a Ppd-Dla) - 34.5 days; haplotype 2 (Vrn-Ala Vrn-B1c vrn-D1 vrn-B3 Ppd-Dla) - 37.3 days; haplotype 3 (Vrn-Ala Vrn-B1c vrn-D1 Vrn-B3a Ppd$D 1 b)$ - 38.0 days; haplotype 4 (Vrn-Ala Vrn-B1c vrn-D1 vrn-B3 Ppd-D1b) - 40.3 days; Tulun 15 (Vrn-Ala Vrn-B1c vrn-D1 Vrn-B3a Ppd-Dla) - 37.5 days; Obskaya 2 (Vrn-Ala $V r n-B 1 c$ vrn-D1 vrn-B3 Ppd-D1b) - 42.9. The results obtained demonstrated that Vrn$B 3 a$ and $P p d-D 1 a$ alleles accelerate heading of plants with the same allelic composition of Vrn-Ala, Vrn-Blc, and vrn-D1 genes in the long day conditions of Novosibirsk region. Acknowledgements: The study has been supported by the Budget project 0324-20190039 . 